\title{
Diagnostic value of interleukin 22 and carcinoembryonic antigen in tuberculous and malignant pleural effusions
}

\author{
DI JIN ${ }^{1,2^{*}}$, YING CHEN $^{1 *}$, ZHENGYUN WANG $^{1}$, SIWEI WANG ${ }^{1}$, HANSVIN BUNJHOO $^{1}$, JING ZHU $^{1}$, \\ YONG CAO $^{1}$, WEINING XIONG ${ }^{1}$, SHENGDAO XIONG ${ }^{1}$, YONGJIAN XU ${ }^{1}$ and HUIJUAN FANG ${ }^{1}$ \\ ${ }^{1}$ Department of Respiratory Disease, Tongji Hospital, Key Lab of Pulmonary Diseases of Health Ministry, \\ Tongji Medical College, Huazhong University of Science and Technology, Wuhan 430030; \\ ${ }^{2}$ Teaching and Research Division of Internal Medicine, Hubei College of Chinese Medicine, Jingzhou 434020, P.R. China
}

Received July 7, 2011; Accepted August 22, 2011

DOI: 10.3892/etm.2011.344

\begin{abstract}
The aim of this study was to investigate the diagnostic value of interleukin 22 (IL-22) and carcinoembryonic antigen (CEA) in tuberculous pleural effusions (TPEs) and malignant pleural effusions (MPEs). Pleural effusion samples from 56 patients were classified on the basis of diagnosis as TPE $(n=28)$ and MPE $(n=28)$. The concentration of IL-22 was determined by ELISA. Lactate dehydrogenase (LDH), adenosine dehydrogenase (ADA) and CEA levels were also determined in all patients. A significant difference was observed in the levels of ADA and CEA $(\mathrm{P}<0.01)$, but not in the levels of LDH $(\mathrm{P}>0.05)$ between TPE and MPE. The concentration of IL-22 in TPE was significantly higher compared to MPE $(\mathrm{P}<0.01)$. With a threshold value of $49 \mathrm{pg} / \mathrm{ml}$, IL-22 had a sensitivity of $82.14 \%$ (23/28) and a specificity of $96.43 \%$ (27/28) for differential diagnosis. The combined detection of IL-22 and CEA had a sensitivity of $100 \%(28 / 28)$ and a specificity of 96.43\% (27/28) to distinguish TPE from MPE. TPEs showed significantly higher levels of IL-22 compared to MPEs. The combined detection of IL-22 and CEA may be more valuable in the differential diagnosis between TPE and MPE.
\end{abstract}

\section{Introduction}

Pleural effusion is one of the most common clinical manifestations of pleural diseases (1-3). According to clinical risk factors and prognosis, pleural effusion can be divided into two categories, benign and malignant (4). The most common cause

Correspondence to: Dr Huijuan Fang, Department of Respiratory Disease, Tongji Hospital, Tongji Medical College, Huazhong University of Science and Technology, 1095 Jiefang Dadao Road, Wuhan 430030, P.R. China

E-mail: fanghuijuan@yahoo.com.cn

*Contributed equally

Key words: interleukin 22, carcinoembryonic antigen, pleural effusion, differential diagnosis of benign pleural effusion is tuberculosis (TB), and that of malignant pleural effusion (MPE) is lung and breast cancer (5). Due to different risk factors and prognosis, it is necessary to differentiate between them. However, this remains a major clinical problem. Although the presence of tumor cells in pleural effusion is a diagnostic marker of MPE, the probability of finding them is low. For cytology-negative pleural effusion, some of the currently used indices, such as lactate dehydrogenase (LDH), adenosine dehydrogenase (ADA) and carcinoembryonic antigen (CEA), have a certain extent of differential value, however, their specificity and sensitivity are limited. Due to the fear of possible trauma caused by thoracoscopy, some patients are not keen to agree on such a procedure. Therefore, searching for new indices is very important.

The improved understanding of pleural effusion immunopathogenesis could lead to the development of new immunodiagnostic tools to facilitate its differential diagnosis. The main cellular components in both tuberculous pleural effusions (TPEs) and MPEs are lymphocytes. Previous research data have reported that lymphocytes play an important regulatory role in the pathogenesis of pleural effusion $(1,2)$. Inflammation leads to the accumulation of lymphocytes in the pleural cavity, which release a variety of mediators and cytokines influencing pleural capillary permeability, resulting in pleural effusion $(6,7)$. Large scale studies have reported that $\mathrm{CD} 4^{+} \mathrm{T}$ lymphocytes play an important role in the pathogenesis of pleural effusion (7). $\mathrm{CD} 4^{+} \mathrm{T}$ cells can be differentiated into Th1, Th2, Th17 and Treg cells. Th17 cells, which form a distinct subset of T helper cells, produce unique cytokines, including interleukin (IL)-17A, IL-17F and IL-22. These cytokines stimulate defensin production and the recruitment of neutrophils and monocytes at the site of inflammation. They are also involved in the early phase of host defence (8-12). Wang et al revealed that Th17 cells can be found in the pleural effusion of patients with $\mathrm{TB}$, suggesting their potential role in immunity against Mycobacterium tuberculosis (13).

Certain research data have shown that Th17 cells are one of the major sources of IL-22. IL-22 is a member of the IL-10 family and is mainly expressed in activated $\mathrm{T}$ and natural killer cells. Its biological targets are epithelial or parenchymal cells in the gut, lungs, skin and kidneys (14-16). In pancreatitis, psoriasis, inflammatory bowel disease, asthma and 
other inflammatory diseases, IL-22 may play an important regulatory role (17-20). Previous studies have reported that in TPE and MPE, the levels of IL-22 are high $(21,22)$. However, whether there is a significant difference in their expression levels, and whether we could distinguish them based on this difference, remains unknown.

Based on the above problems, the idea was to collect samples of TPE and MPE, determine the expression of IL-22 by ELISA, and explore its value in the differential diagnosis between TPE and MPE.

\section{Materials and methods}

Pleural effusion samples. In this study, samples of pleural effusions were collected from 56 patients who were hospitalized in the Department of Respiratory Medicine, Tongji Hospital Affiliated to Tongji Medical College, Huazhong University of Science and Technology, between April 2009 and May 2011. Written consent was obtained from all the patients concerned in order to perform this study. Pleural effusions were divided into TPE (28 cases; 24 males and 4 females; $41.46 \pm 3.34$ years of age) and MPE groups (28 cases; 10 males and 14 females; $58.71 \pm 2.1$ years of age).

Diagnostic criteria for pleural effusions. The pleural effusions were firstly diagnosed as exudates using Light's criteria. The diagnostic criteria for MPE were: Cytological evidence of malignant cells present in pleural effusion or from biopsies taken. TPE was diagnosed according to the following principle: Identification of M. tuberculosis, pleural biopsy revealing granulomatous tissue, positive PPD test and positive response to anti-TB treatment.

Samples collection. Pleural effusions were collected before any treatment was initiated within $24 \mathrm{~h}$ after hospitalization. Some of the pleural fluids were sent to the hospital laboratory to detect levels of total protein (Pro), LDH, ADA and CEA. Some other pleural effusions $(100 \mathrm{ml})$ were centrifuged at $4^{\circ} \mathrm{C}$ $1200 \mathrm{r} / \mathrm{min}$ for $15 \mathrm{~min}$, and the supernatants were immediately frozen with $500 \mu \mathrm{l}$ Ep tubes at $-80^{\circ} \mathrm{C}$.

Measurement of IL-22. The concentration of IL-22 in pleural effusions was measured by the enzyme-linked immunosorbent assay kit (ELISA) according to the manufacturer's protocol (Bender, Austrilian). All samples were assayed in duplicate.

Statistical analysis. Data were expressed as the means \pm SEM. Difference in data was analyzed by the Student's t-test or the $\chi^{2}$ test, using receiver operating curve (ROC) analysis to evaluate the threshold value of IL-22 and CEA in differentiating TPE from MPE. For each ROC, a cut-off point was determined as the value of the parameter that maximized the sum of specificity and sensitivity. A value of $\mathrm{P}<0.05$ was considered significant. Statistical analysis was carried out using SPSS 17.0 software.

\section{Results}

General characteristics of the pleural effusions. Significant differences were observed in age, as well as CEA and ADA
Table I. Descriptive statistics of each pleural effusion group.

\begin{tabular}{lcc}
\hline Groups & TPE $(\mathrm{n}=28)$ & MPE $(\mathrm{n}=28)$ \\
\hline Age & $41.46 \pm 3.34^{\mathrm{a}}$ & $58.71 \pm 2.10$ \\
Gender (male/female) & $24 / 4$ & $10 / 18$ \\
CEA (ng/ml) & $1.29 \pm 0.11^{\mathrm{a}}$ & $482.86 \pm 115.26$ \\
Pro $(\mathrm{g} / \mathrm{l})$ & $48.47 \pm 1.52$ & $44.14 \pm 1.71$ \\
LDH (U/l) & $317.86 \pm 36.54$ & $557.25 \pm 117.35$ \\
ADA (IU/l) & $54.75 \pm 5.03^{\mathrm{a}}$ & $23.45 \pm 6.40$
\end{tabular}

TPE, tuberculous pleural effusions; MPE, malignant pleural effusions; CEA, carcinoembryonic antigen; Pro, protein; LDH, lactate dehydrogenase; ADA, adenosine dehydrogenase. Data are as presented as the means $\pm \mathrm{SEM},{ }^{\mathrm{a}} \mathrm{P}<0.01$.

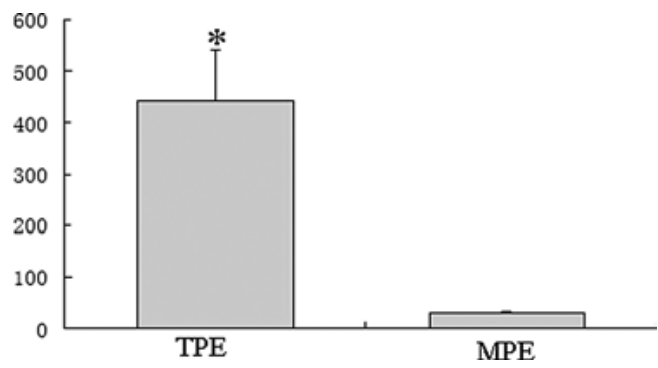

Figure 1. Concentration of IL-22 in TPE and MPE. Data are presented as the means \pm SEM and the value between the TPE and MPE groups differs significantly. ${ }^{*} \mathrm{P}<0.01$

levels in TPE and MPE $(\mathrm{P}<0.01)$. We did not find a significant difference in the concentration of Pro and LDH between TPE and MPE $(\mathrm{P}>0.05$, Table I).

IL-22 concentration in pleural effusions. As shown in Fig. 1, the concentration of IL-22 in the TPE group (441.91 $\pm 99.34 \mathrm{pg} /$ ml) was significantly higher compared to the MPE group (29.81 $\pm 2.15 \mathrm{pg} / \mathrm{ml} ; \mathrm{P}<0.01)$.

Diagnostic value of IL-22 in TPE and MPE. The diagnostic threshold afforded by the ROC analysis for IL-22 was 49 pg/ $\mathrm{ml}$ (Fig. 2). The area under the IL-22 ROC was 0.902. Using a threshold value of $49 \mathrm{pg} / \mathrm{ml}$, IL-22 had a sensitivity of $82.14 \%$ (23/28), a specificity of $96.43 \%$ (27/28), an accuracy of $89.29 \%(50 / 56)$, a positive predictive value of $95.8 \%$ $(23 / 24)$ and a negative predictive value of $84.4 \%(27 / 32)$ (Fig. 3).

Diagnostic value of combined detection of IL-22 and CEA in TPE and MPE. Firstly, CEA levels were detected and the diagnostic value in TPE and MPE was analyzed. The diagnostic threshold afforded by the ROC analysis for CEA was $2.42 \mathrm{ng} / \mathrm{ml}$. The area under the CEA ROC was 0.935. It was higher compared to the areas of IL-22 (Fig. 4). With a threshold value of $2.42 \mathrm{ng} / \mathrm{ml}, \mathrm{CEA}$ had a sensitivity of $89.3 \%(25 / 28)$, a specificity of $96.43 \%(27 / 28)$, an accuracy of $92.86 \%(52 / 56)$, a positive predictive value of $96.15 \%(25 / 26)$ and a negative 


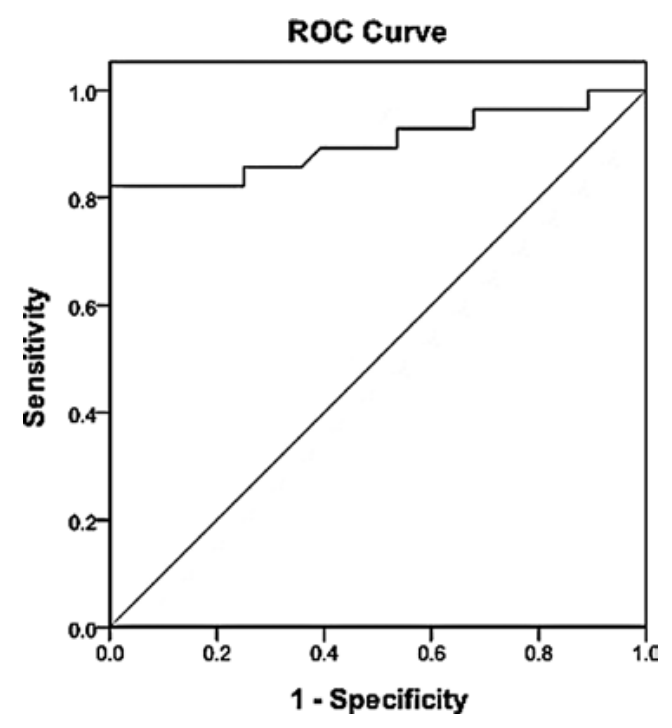

Figure 2. ROC of IL-22, for the diagnosis of TPE vs. MPE.

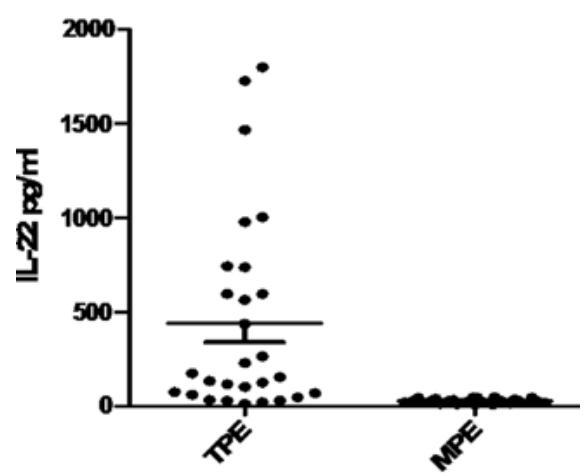

Figure 3. IL-22 levels in TPE and MPE. Cut-off points were $49 \mathrm{pg} / \mathrm{ml}$.

predictive value of 90\% (27/30) (Fig. 5). The sensitivity of IL-22 was lower compared to CEA. Between the studied parameters, IL-22 and CEA, no significant differences were found with respect to the specificity.

The combined diagnostic value of IL-22 and CEA in TPE and MPE was further detected. The results showed that the combined detection of these two indices had a sensitivity of $100 \%(28 / 28)$ and a specificity of $96.43 \%$ (27/28). The sensitivity was higher compared to the two separate tests for TPE and MPE. However, no significant differences were found with respect to specificity.

\section{Discussion}

In this study, the concentration of IL-22 was significantly increased in TPE and MPE, similar to results from other studies (22). Simultaneously, we found that IL-22 levels were significantly higher in TPE compared to MPE, suggesting more pleural sources of IL-22 in TB patients and a high differential diagnostic value of IL-22 among TPE and MPE. Using the combined detection of IL-22 and CEA, the diagnostic value was more accurate compared to the single index.

Clinically, the presence of tumor cells in pleural effusion is the gold standard for MPE. Nevertheless, for cytology-negative

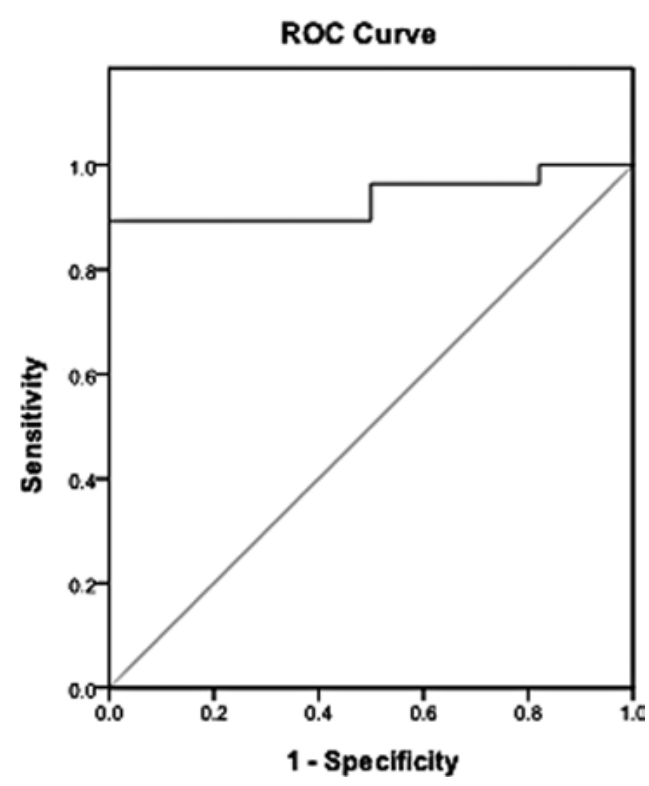

Figure 4. ROC of CEA, for the diagnosis of TPE vs. MPE.

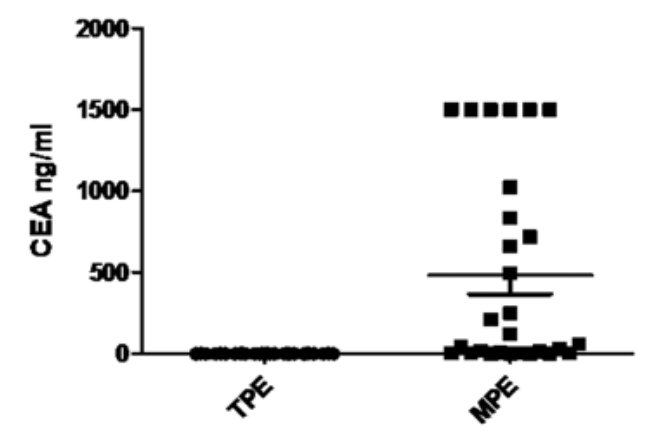

Figure 5. CEA levels in TPE and MPE. Cut-off points were $2.42 \mathrm{ng} / \mathrm{ml}$.

effusion, the main problem was to differentiate benign pleural effusion from MPE and to decide on further treatment. In our results, we found that certain indices, such as LDH and Pro in pleural effusion had no significant differential values. However, CEA and IL-22 expression in TPE and MPE was significantly different, which might be helpful in distinguishing between them.

Reports regarding the association of IL-22 expression with pleural effusions are few and varied. Qiao et al reported that the expression of IL-22 was significantly increased in TPE (21). Zhang et al also demonstrated this view. However, they found that IL-22 levels in MPE were higher compared to TPE (22). This differs from our results. Such a contradiction can be justified and explained by the fact that more pleural effusion samples were collected and used in our study.

IL-22 was found in T lymphocytoma by Dumoutier et al in 1999 (23). IL-22 belongs to IL-10-related cytokines, whose receptor complex consists of two chains, IL-22R1 and IL-10R2. Aujla et al demonstrated that IL-22 is a crucial immune mediator produced by $\mathrm{T}$ cells and a critical mediator in mucosal host defense (24). Previous studies have reported that IL-22 can induce the synthesis of acute phase proteins in acute hepatitis and pancreatitis $(25,17)$. Other data have demonstrated that IL-22 can also play an important regula- 
tory role in the process of asthma and allergic diseases (26). In addition, IL-22 is also involved in the pathogenesis of autoimmune diseases. Ikeuchi et al found that IL-22 promoted the proliferation of synovial fibroblasts and increased the secretion of various inflammatory mediators and cytokines, which participate in the pathogenesis of rheumatoid arthritis (27). Schmechel et al found that IL-22 serum levels in Crohn's patients were significantly higher compared to the normal controls and closely related with disease activity (19). Wolk et al found that the expression of IL-22 was unusually high in psoriatics patients (18).

In this study, IL-22 expression was significantly higher in TPE when compared to MPE, indicating the involvement of IL-22 in the pathogenesis of pleural effusion. However, the specific mechanism of IL-22 in the pathogenesis of pleural effusion was unclear. Th17 cells are a major source of IL-22. In addition to the traditional Th1 and Th2 subsets, Th17 cells belong to a recently identified T helper subset. Th17 cells play an important regulatory role in the pathogenesis of infections and autoimmune diseases by releasing IL-17A, IL-17F, IL-22 and other cytokines (16). Therefore, we presumed that acute or chronic inflammation causes the accumulation of lymphocytes which release a variety of inflammatory mediators and cytokines such as IL-22, increasing the pleural capillary permeability, thus resulting in pleural effusion.

Clinically, although lymphocytic cellular components are the base of TPE and MPE, the lymphocyte subsets are different. This causes a difference in the concentration of inflammatory mediators and cytokines and may explain the higher expression of IL-22 in TPE found in our study. However the specific role of IL-22 in the pathogenesis of TPE and MPE requires further research.

CEA has been studied extensively and has been found to have a differential value in distinguishing benign pleural effusion from MPE. Boucher et al reported that CEA concentration in malignant tissues was on average 60-fold higher compared to the non-malignant tissues (28). CEA was one of the most commonly used clinically identified indices in benign pleural effusion and MPE. In our study, the diagnostic value of CEA in TPE and MPE was $2.42 \mathrm{ng} / \mathrm{ml}$. The threshold value of the diagnosis of TPE and MPE had a sensitivity of $89.3 \%$ (25/28) and a specificity of (27/28). These results are consistent with those from the study by Shi et al (29). In particular, we discovered that the combined IL-22 and CEA detection had a sensitivity of $100 \%(28 / 28)$ and a specificity of $96.43 \%(27 / 28)$. The diagnostic value was higher compared to the single index. These results may provide a new approach with a higher diagnostic value in TPE and MPE.

In summary, our results suggest that IL-22 is higher in TPE compared to MPE. The critical value of IL-22 protein for diagnosing TPE and MPE was $49 \mathrm{pg} / \mathrm{ml}$. The combined detection of CEA and IL-22 had a higher sensitivity compared to the single index. This may effectively improve the diagnostic performance.

\section{Acknowledgements}

This study was supported in part by a research grant from the Hubei Programs for Science and Technology Development (2002AA301D11).

\section{References}

1. Doelken P: Clinical implications of unexplainable lung due to pleural disease. Am J Med Sci 335: 21-25, 2008.

2. Khaleeq $\mathrm{G}$ and Musani AI: Emerging paradigms in the management of malignant pleural effusions. Respir Med 102: 939-948, 2008.

3. Musani AI: Treatment options for malignant pleural effusion. Curr Opin Pulm Med 15: 380-387, 2009.

4. Spector M and Pollak JS: Management of malignant pleural effusions. Semin Respir Crit Care Med 29: 405-413, 2008.

5. Heffner JE: Diagnosis and management of malignant pleural effusions. Respirology 13: 5-20, 2008.

6. Yang HB and Shi HZ: T lymphocytes in pleural effusion. Chin Med J (Engl) 121: 579-580, 2008.

7. Qin XJ, Shi HZ, Liang QL, Huang LY and Yang HB: $\mathrm{CD} 4^{+} \mathrm{CD} 25^{+}$ regulatory $\mathrm{T}$ lymphocytes in tuberculous pleural effusion. Chin Med J (Engl) 121: 581-586, 2008.

8. Infante-Duarte C, Horton HF, Byrne MC and Kamradt T: Microbial lipopeptides induce the production of IL17 in Th cells. J Immunol 165: 6107-6115, 2000

9. Park H, Li Z, Yang XO, Chang SH, Nurieva R, Wang YH, Wang Y, Hood L, Zhu Z, Tian Q and Dong C: A distinct lineage of $\mathrm{CD} 4^{+} \mathrm{T}$ cells regulates tissue inflammation by producing interleukin 17. Nat Immunol 6: 1133-1141, 2005.

10. Harrington LE, Hatton RD, Mangan PR, Turner H, Murphy TL, Murphy KM and Weaver CT: Interleukin17-producing CD4 ${ }^{+}$ effector $\mathrm{T}$ cells develop via a lineage distinct from the $\mathrm{T}$ helper type 1 and 2 lineages. Nat Immunol 6: 1123-1133, 2005.

11. Harrington LE, Mangan PR and Weaver CT: Expanding the effector CD4 T-cell repertoire: the Th17 lineage. Curr Opin Immunol 18: 349-356, 2006.

12. Weaver CT, Harrington LE, Mangan PR, Gavrieli M and Murphy KM: Th17: an effector CD4 T cell lineage with regulatory T cell ties. Immunity 24: 677-688, 2006.

13. Wang T, Lv M, Qian Q, Nie Y, Yu L and Hou Y: Increased frequencies of $\mathrm{T}$ helper type 17 cells in tuberculous pleural effusion. Tuberculosis 91: 231-237, 2011

14. Wolk K, Witte E, Witte K, Warszawska K and Sabat R: Biology of interleukin-22. Semin Immunopathol 32: 17-31, 2010.

15. Dumoutier L, Lejeune D, Colau D and Renauld JC: Cloning and characterization of IL-22 binding protein, a natural antagonist of IL-10-related T cell-derived inducible factor/IL-22. J Immunol 166: 7090-7095, 2001.

16. Wolk K and Sabat R: Interleukin-22: a novel T and NK cellderived cytokine that regulates the biology of tissue cells. Cytokine Growth Factor Rev 17: 367-380, 2006.

17. Aggarwal S, Xie MH, Maruoka M, Foster J and Gurney AL: Acinar cells of the pancreas are a target of interleukin-22. J Interferon Cytokine Res 21: 1047-1053, 2001.

18. Wolk K, Witte E, Wallace E, Döcke WD, Kunz S, Asadullah K, Volk HD, Sterry W and Sabat R: IL-22 regulates the expression of genes responsible for antimicrobial defense, cellular differentiation, and mobility in keratinocytes: a potential role in psoriasis. Eur J Immunol 36: 1309-1323, 2006.

19. Schmechel S, Konrad A, Diegelmann J, Glas J, Wetzke M, Paschos E, Lohse P, Göke B and Brand S: Linking genetic susceptibility to Crohn's disease with Th17 cell function: IL-22 serum levels are increased in Crohn's disease and correlate with disease activity and IL23R genotype status. Inflamm Bowel Dis 14: 204-212, 2008.

20. Schnyder B, Lima C and Schnyder-Candrian S: Interleukin-22 is a negative regulator of the allergic response. Cytokine 50: 220-227, 2010.

21. Qiao D, Yang BY, Li L, Ma JJ, Zhang XL, Lao SH and Wu CY: ESAT-6- and CFP-10-specific Th1, Th22 and Th17 cells in tuberculous pleurisy may contribute to the local immune response against Mycobacterium tuberculosis infection. Scand J Immunol 73: 330-337, 2011

22. Zhang W, Chen Y, Wei H, Zheng C, Sun R, Zhang J and Tian Z: Antiapoptotic activity of autocrine interleukin-22 and therapeutic effects of interleukin-22-small interfering RNA on human lung cancer xenografts. Clin Cancer Res 14: 6432-6439, 2008.

23. Dumoutier L, Lejeune D, Colau D and Renauld JC: Cloning and characterization of IL-10 related $\mathrm{T}$ cell derived inducible factor (IL-TIF), a novel cytokine structurally related to IL-10 and inducible by IL-9. J Immunol 164: 1814-1819, 2000.

24. Aujla SJ and Kolls JK: IL-22: a critical mediator in mucosal host defense. J Mol Med1 87: 451-454, 2009. 
25. Dumoutier L, van Roost E, Colau D and Renauld JC: Human interleukin-10-related T cell-derived inducible factor: molecular cloning and functional characterization as a hepatocyte-stimulating factor. Proc Natl Acad Sci USA 97: 10144-10149, 2000.

26. Kotenko SV, Izotova LS, Mirochnitchenko OV, Esterova E, Dickensheets H, Donnelly RP and Pestka S: Identification of the functional interleukin-22 (IL-22) receptor complex: the IL-10R2 chain (IL-10Rbeta) is a common chain of both the IL-10 and IL-22 (IL-10-related T cell-derived inducible factor, IL-TIF) receptor complexes. J Biol Chem 276: 2725-2732, 2001.
27. Ikeuchi H, Kuroiwa T, Hiramatsu N, Kaneko Y, Hiromura K, Ueki K and Nojima Y: Expression of interleukin-22 in rheumatoid arthritis: potential role as a proinflammatory cytokine. Arthritis Rheum 52: 1037-1046, 2005.

28. Boucher D, Cournoyer D, Stanners CP and Fuks A: Studies on the control of gene expression of the carcinoembryonic antigen family in human tissue. Cancer Res 49: 847-852, 1989.

29. Shi HZ, Liang QL, Jiang J, Qin XJ and Yang HB: Diagnostic value of carcinoembryonic antigen in malignant pleural effusion: a meta-analysis. Respirology 13: 518-527, 2008. 